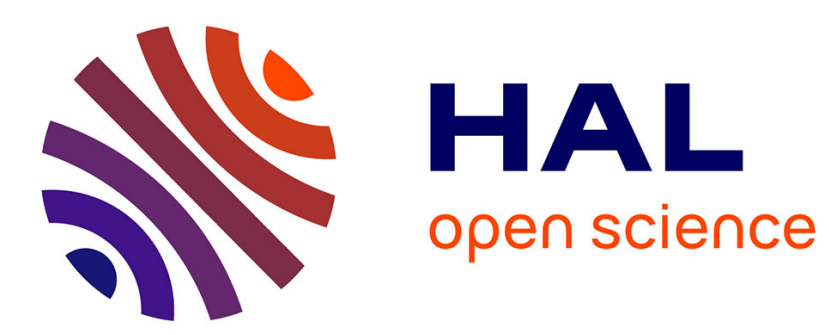

\title{
SEMICLASSICAL CALCULATIONS OF THE IMAGINARY PART OF THE NUCLEON-NUCLEUS OPTICAL POTENTIAL
}

R. Hasse, P. Schuck

\section{> To cite this version:}

R. Hasse, P. Schuck. SEMICLASSICAL CALCULATIONS OF THE IMAGINARY PART OF THE NUCLEON-NUCLEUS OPTICAL POTENTIAL. Workshop on Semiclassical Methods in Nuclear Physics, 1984, Grenoble, France. pp.C6-213-C6-220, 10.1051/jphyscol:1984625 . jpa-00224227

\section{HAL Id: jpa-00224227 https://hal.science/jpa-00224227}

Submitted on 1 Jan 1984

HAL is a multi-disciplinary open access archive for the deposit and dissemination of scientific research documents, whether they are published or not. The documents may come from teaching and research institutions in France or abroad, or from public or private research centers.
L'archive ouverte pluridisciplinaire HAL, est destinée au dépôt et à la diffusion de documents scientifiques de niveau recherche, publiés ou non, émanant des établissements d'enseignement et de recherche français ou étrangers, des laboratoires publics ou privés. 


\author{
R.W. Hasse and P. Schuck \\ Institut Laue-Langevin, 38042 Grenoble Cedex, France \\ * Institut des Sciences Nucléaires, 38026 Grenoble Cedex, France
}

\begin{abstract}
Rêsumé - On calcule pour des noyaux finis, la partie imaginaire du potentiel optique noyau-nucléon sur et hors couche utilisant l'approximation du gaz de Fermi local et une force d'échange à deux corps ayant une portée finie. on compare les résultats avec ceux obtenus par des calculs pour la matière infinie et ceux obtenus soit pour la densité locale soit pour l'approximation de Glauber.

Abstract - We calculate for finite nuclei the imaginary part of the nucleusnucleon optical potential on and off shell by using the local Fermi gas approximation and a finite range two-body exchange force. Results are compared with those obtained by infinite nuclear matter calculations as well as using the local density or Glauber approximation.
\end{abstract}

I - INTRODUCTION

The imaginary part of the nucleus-nucleon optical potential enters directly in various quantities of interest in static properties of nuclei or in nuclear reaction theories, e.g. in the effective mass /1/ and the self energy /2-5/, the nucleon mean free path /6-7/and quasi-particle life times /8/ or in theories of inelastic scattering. Whereas the optical potential has been often calculated fully microscopically, semiclassical calculations exist only for infinite nuclear matter $/ 3,9,10 /$ or finite nuclei with the local density approximation /5/. The latter approximation consists of replacing the Fermi energy $\lambda$ by its local equivalent $\varepsilon_{F}(R)$. A review over existing semiclassical calculations is given in ref. /11/.

In this paper we, are concerned with finite nuclei and take the finite size effects into account exactly with help of an average nuclear potential $V(R)$ which we need not specify explicitly. Furthermore, we also calculate the off shell behaviour of the optical potential which has often been assumed to be weak/7/. In order to be realistic, we employ a finite range exchange potential which forces the optical potential to decrease at high energies. Results will also be compared with those obtained by use of the Glauber approximation which neglects the Pauli principle. Preliminary results have already been presented in refs. $/ 12,13 /$.

\title{
II - THE MODEL
}

Beyond Hartree-Fock, the first corrections to the nuclear self energy are given by core polarization and correlation contributions according to Fig. 1 .
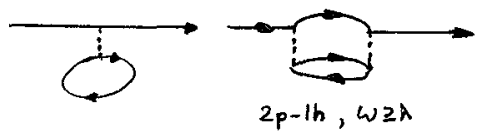

Hartree-Fock

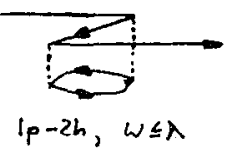

Correlation
Fig. 1 - The first three graphs of the nuclear self energy. 
Written in terms of the mass operator,

$$
M_{141}^{w}=\frac{1}{2} \bar{v}_{1234}\left\{\frac{h_{2} \bar{n}_{3} \bar{n}_{4}}{\omega+\varepsilon_{2}-\varepsilon_{3}-\varepsilon_{4}+i \eta}-\frac{\bar{h}_{2} h_{3} h_{4}}{\omega+\varepsilon_{2}-\varepsilon_{3}-\varepsilon_{4}-i \eta}\right\} \bar{v}_{4321^{\prime}}
$$

where $\bar{n}$, $n$ and $\varepsilon$ are the particle and hole densities and energies, respectively, and $\bar{v}$ is the two-body interaction, one sees that the polarization graph contributes only if the energy $\omega$ is above the Fermi energy $\lambda$ and the correlation graph only if $\omega$ is smaller than $\lambda$. Rewriting the imaginary part of (1) in space representation, one obtains the nonfocal imaginary part of the optical potential,

$$
\begin{aligned}
W_{r r^{\prime}}^{\omega}= & \frac{\pi}{2} \int_{-\infty}^{+\infty} \frac{d t}{2 \pi} e^{i \omega t} \int d^{3} r_{1} d^{3} r_{2} d^{3} r_{3} d^{3} r_{3}^{\prime} d^{3} r_{2}^{\prime} d^{3} r_{1}^{\prime} \\
& \times \bar{v}\left(r r_{1} r_{2} r_{3}\right) \bar{v}\left(r_{3}^{\prime} r_{2}^{\prime} r_{1}^{\prime} r^{\prime}\right) \\
& \times\left[\left\langle r_{1}\left|\rho e^{i H t}\right| r_{1}^{\prime}\right\rangle\left\langle r_{2}\left|\rho e^{-i H t}\right| r_{2}^{\prime}\right\rangle\left\langle r_{3}\left|\rho e^{-i H t}\right| r_{3}^{\prime}\right\rangle\right. \\
& \left.+\left\langle r_{1}\left|\bar{\rho} e^{-i H t}\right| r_{1}^{\prime}\right\rangle\left\langle r_{2}\left|\rho e^{i H t}\right| r_{2}^{\prime}\right\rangle\left\langle r_{3}\left|\rho e^{i H t}\right| r_{3}^{\prime}\right\rangle\right] .
\end{aligned}
$$

Here $\bar{\rho}, \rho$ and $H$ are the particle and hole density operators and the one particle Hamiltonian, respectively.

In order to obtain the coordinate and momentum dependent optical potential for a contact exchange force

$$
\bar{v}\left(r r_{1} r_{2} r_{3}\right)=\bar{v}\left(\mid \vec{r}-\vec{r}_{1}\right) \delta\left(\vec{r}-\vec{r}_{2}\right) \delta\left(\vec{r}_{1}-\vec{r}_{3}\right),
$$

we go over to relative and center-of-mass coordinates according to

$$
\vec{s}=\vec{\gamma}-\vec{\gamma}^{\prime} \quad \vec{R}=\frac{\vec{\gamma}+\vec{\gamma}^{\prime}}{2}
$$

and Fourier transform with respect to the c.m. coordinates, $\vec{s} \rightarrow \vec{p}, \vec{s}_{i} \rightarrow \vec{p}_{i}$. Then the matrix elements in eq. (2) can be written in semiclassical approximation as

$$
\left\langle\vec{r}\left|g e^{i H t}\right| \vec{r}\right\rangle=\int \frac{d^{3} p}{(2 \pi)^{3}} e^{i \vec{p} \vec{s}} e^{i t\left(\frac{p^{2}}{2 m}+V(R)\right)} \theta\left(\lambda-\frac{p^{2}}{2 m}-V(R)\right)
$$

and, similarly, for $\vec{p} \exp (-i H t)$. This yields

$$
\begin{aligned}
W(\omega, P, R) & =\frac{\pi}{2} \int \frac{d t}{2 \pi} \int d^{3} r_{1} \int d^{3} r_{1}^{\prime} v\left(\left|\vec{r}-\vec{r}_{1}\right|\right) v\left(\left|\vec{r}^{\prime}-\vec{r}_{1}^{\prime}\right|\right) \int d^{3} s e^{i \vec{s} \vec{P}} \\
& \times \int \frac{d^{3} P_{1}^{-\infty}}{(2 \pi)^{3}} \int \frac{d^{3} P_{2}}{(2 \pi)^{3}} \int \frac{d^{3} P_{3}}{(2 \pi)^{3}} e^{i\left(\vec{P}_{1} \vec{s}_{4}-\vec{P}_{2} \vec{s}-\vec{P}_{3} \vec{s}_{1}\right)} e^{i t\left(\omega+\frac{p_{1}^{2}-P_{2}^{2}-P_{3}^{2}}{2 m}-V(R)\right)} \\
& \times\left[\theta\left(\lambda-\frac{P_{1}^{2}}{2 m}-V\left(R_{1}\right)\right) \theta\left(\frac{P_{2}^{2}}{2 m}+V(R)-\lambda\right) \theta\left(\frac{P_{3}^{2}}{2 m}+V\left(R_{1}\right)-\lambda\right)+\theta_{-} \theta_{-} \theta_{-}\right],
\end{aligned}
$$

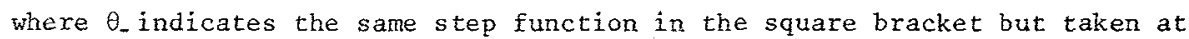
negative argument. Now the time and relative coordinate integrationscan be performed to give 


$$
\begin{aligned}
& W(\omega, P, R)=\frac{2}{(2 \pi)^{5}} \int d^{3} R_{1} \int d^{3} P_{1} \int d^{3} P_{2} \int \alpha^{3} P_{3} \\
& \times\left[\theta\left(p_{F}^{2}\left(R_{1}\right)-p_{1}^{2}\right) \theta\left(p_{2}^{2}-p_{f}^{2}(R)\right) \theta\left(p_{3}^{2}-P_{f}^{2}\left(R_{n}\right)\right)+\theta_{-} \theta_{-} \theta_{-}\right] \\
& \times \delta\left(\vec{P}_{+}+\vec{p}_{1}-\vec{p}_{2}-\vec{p}_{3}\right) \delta\left(\omega-\lambda+\varepsilon_{f}(R)+\left(p_{1}^{2}-p_{2}^{2}-P_{3}^{2}\right) / 2 m\right) \\
& \times \int d^{3} \times e^{2 i\left(\vec{P}-\vec{P}_{2}\right) \vec{x}} v\left(\left|\vec{R}-\overrightarrow{R_{1}}+\vec{x}\right|\right) v\left(\left|\vec{R}-\vec{R}_{1}-\vec{x}\right|\right) \text {, }
\end{aligned}
$$

where we have introduced the local Fermi energy and the local Fermi momentum according to

$$
\varepsilon_{F}(R)=\frac{P_{F}^{2}(R)}{2 m}:=\lambda-V(R) \text {. }
$$

In eq. (7) finite size effects enter directly in the energy conserving $\delta$-function by the additional local Fermi energy.

For simplicity, we now use a Gaussian force of range $r_{0}$ in coordinate or of range $\mathrm{k}_{\mathrm{o}}$ in momentum space

$$
\begin{aligned}
& \bar{v}(r)=v_{0} e^{-\left(r / r_{0}\right)^{2}} \\
& \bar{v}(k)=\frac{v_{0}}{2 \pi^{5 / 2} k_{0}^{3}} e^{-\left(k / k_{0}\right)^{2}}, \quad k_{0}=2 / r_{0} .
\end{aligned}
$$

Then the integration over $\vec{x}$ in eq. (7) can be performed. Furthermore, the momentum conserving $\delta$-function suggests to introduce the relative and $c . m$. momentum transfers according to

$$
\begin{aligned}
& \vec{p}_{1}=\vec{q}-\vec{q} / 2 \\
& \vec{P}_{2}=\vec{p}+\vec{q} / 2 \\
& \vec{p}_{3}=\vec{p}-\vec{q}
\end{aligned}
$$

and to use the angle convention

$$
\begin{aligned}
& \vec{p} \cdot \vec{q}=p_{z} q \\
& p^{2}=p_{\perp}^{2}+p_{z}^{2} \\
& \vec{p} \cdot \vec{q}=P_{q} \hat{q}
\end{aligned}
$$

Then the $\hat{q}$ and $p_{\mathcal{L}}$ integrations can be performed to yield the final result

$$
\begin{aligned}
& W(\omega, P, R) \propto \\
& \frac{1}{R} \int_{0}^{R_{0}} \alpha R^{\prime} R^{\prime}\left[e^{-k_{0}^{2}\left(R-R^{\prime}\right)^{2} / 2}-e^{-k_{0}^{2}\left(R+R^{\prime}\right)^{2} / 2}\right] \\
& \times \frac{1}{2 P} \int d p_{z} \int d q q e^{-2 q^{2} / K_{0}^{2}}\left[2 p_{z} \theta \theta\left(p_{F}\left(R^{\prime}\right)-p_{z}-\frac{q}{2}\right)+\left(p_{F}^{2}\left(R^{\prime}\right)-\left(p_{z}-\frac{q}{2}\right)^{2}\right) \theta\left(p_{z}+\frac{q}{2}-p_{F}\left(R^{\prime}\right)\right)\right] \\
& \times \theta\left(2 m|w-\lambda|-2 p_{z} q\right) \theta\left(p_{f}\left(R^{\prime}\right)-\left|p_{z}-\frac{q}{2}\right|\right) \theta\left(2 p_{q}-\left|q^{2}+2 p_{z} q+p^{2}-2 m\left(w-\lambda+\varepsilon_{f}(R)\right)\right|\right) \\
& \mp \text { for } \omega \leqslant \lambda \text {, }
\end{aligned}
$$


where $R_{o}$ is the turning point at which $v\left(R_{0}\right)=\lambda$.

\section{III - ZERO RANGE FORCE}

Eq. (12) simplifies considerably if the range of the force tends to zero or $k \rightarrow \infty$. The first line of eq. (12) becomes constant and the second and third lines reduce to

$$
W(\omega, P, R) \propto \frac{1}{2 R} \int d_{p_{z}} \int d q q \times\left\{\begin{array}{l}
2 p_{z} q \\
p_{F}^{2}(R)-\left(p_{z}-q / 2\right)^{2}
\end{array} \text { for } p_{z}+\frac{q}{2}\left\{\begin{array}{l}
\leq p_{F}(R) \\
\geq p_{F}(R)
\end{array}\right.\right.
$$

$$
\begin{aligned}
& 2 p_{z} q \leq 2 m|\lambda-\omega|, \quad\left|p_{z}-\frac{q}{2}\right| \leq p_{f}(R) \\
& \left|q^{2} F^{2} p_{z} q+q^{2}-2 m\left(\omega-\lambda+\varepsilon_{f}(R)\right)\right| \leq 2 p_{q} \quad \text { for } \omega \leq \lambda .
\end{aligned}
$$

The dependence of $W$ on $R$ here is onty contained in the local Fermi energy (8) so that eq. (13) can be employed for arbitrary potentials. Here and in the following we normalize $W$ to attain the value $1 / 4 \lambda$ at $\omega=P=R=0$. Momentum transfers are limited to

$$
0 \leq P \leq \begin{cases}2 p_{f}(R)+\sqrt{2 m\left(\lambda-\omega+\varepsilon_{F}(R)\right)} & \omega \leq \lambda \\ P_{f}(R)+2 \sqrt{2 m\left(\omega-\lambda+\varepsilon_{f}(R)\right)} & \omega \geq \lambda,\end{cases}
$$

which provides also a small energy cutoff.

Results for $R=0$ are shown in Figs. 1 and 2 . One observes the well known quadratic behaviour around $\omega=\lambda$ which persists even for large $P$ and which is fairly independent of $P$. However, contrary to the claims in refs. $/ 2,8 /$, contributions from the polarization and from the correlation graphs are not strictly symmetric. This effect is strongly pronounced at small P. As a consequence of infinite quasiparticle lifetime at $\omega=\lambda, W(\omega=\lambda, P, R)$ must vanish. Finally, one observes that the nonloca1ity of $W$ is rather important for all energies except $\omega \approx \lambda$, which calls in question the often made "innocous assumption" /7/ of a local imaginary part of the optical potential.

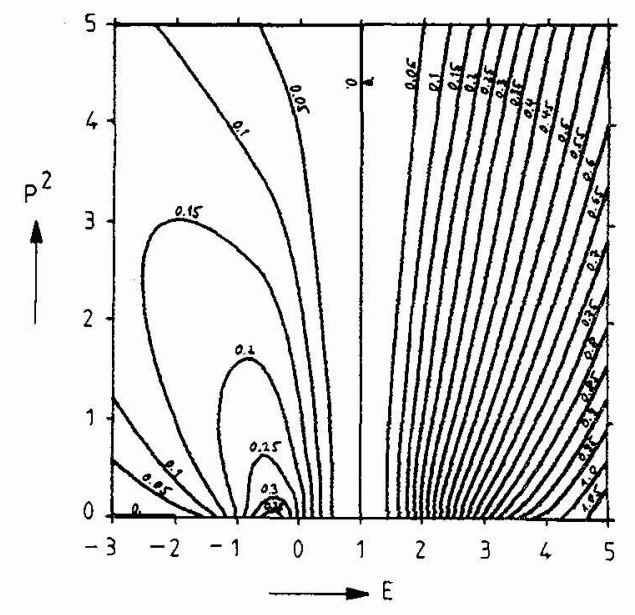

Fig. 1 - Contour plot of $W(\omega, P, R=0)$ for a zero range force. Energy $E$ and energy transfer $\mathrm{P}^{\frac{1}{2}} / 2 \mathrm{~m}$ are measured in units of the Fermi energy.

For zero momentum transfer, $W(\omega, \mathrm{P}=0, \mathrm{R}=0$ ) can be calculated analytically $(\varepsilon=\omega / \lambda)$, 


$$
W(\omega, P=0, R=0) \propto \frac{1}{4} \begin{cases}2-\frac{1}{2} \varepsilon^{2}+\varepsilon^{2} \log \frac{|\varepsilon|}{2} & -2 \lambda \leq \omega \leq-\lambda / 2 \\ 2-\frac{1}{2} \varepsilon^{2}-(1+\varepsilon) \sqrt{1+2 \varepsilon}+\varepsilon^{2} \log \frac{|\varepsilon|(1+\sqrt{1+2 \varepsilon})}{2(1-\sqrt{1+2 \varepsilon})} & -\lambda / 2 \leq \omega \leq 0 \\ (\varepsilon-1)^{2} & 0 \leq \omega \leq 2 \lambda \\ \frac{3}{2} \varepsilon^{2}-2 \varepsilon-1-\varepsilon^{2} \log \frac{\varepsilon}{2} & 2 \lambda \leq \omega \leq 4 \lambda \\ (1+\varepsilon) \sqrt{1+2 \varepsilon}-\varepsilon^{2} \log \frac{\sqrt{1+2 \varepsilon}+1}{\sqrt{1+2 \varepsilon}-1} & 4 \lambda \leq \omega<\infty,\end{cases}
$$

which provides a good check of numerical accuracy. This quantity, together with the on shell value $\left(P^{2} / 2 m=\omega\right)$, the $P$-integrated local equivalent of the nonlocal $W$,

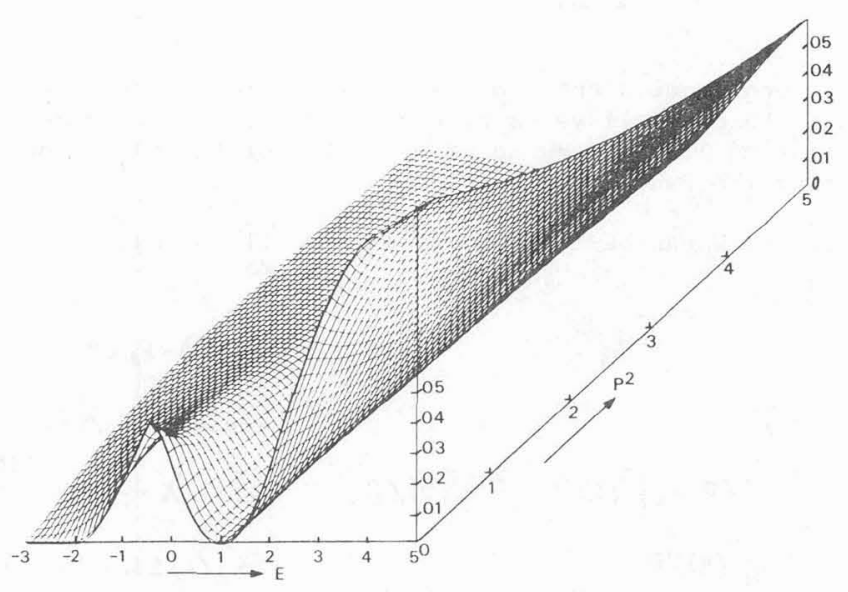

Fig. $2 a$ and $2 b$ - Same as Fig. 1, but three dimensional (front and back views).

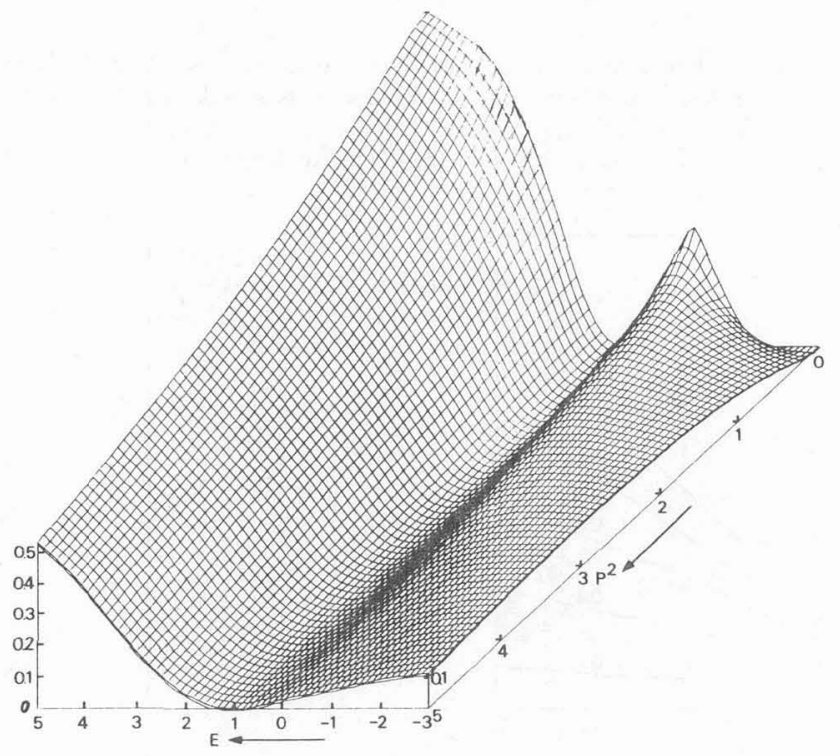


and the Glauber approximation are shown in Fig. 3. Here the Glauber result has been obtained by neglecting the Pauli principle, i.e. dropping the second and third $\theta-$ functions in eq. (7) and neglecting the hole energy $p_{1}^{2} / 2 \mathrm{~m}$ in the energy conserving

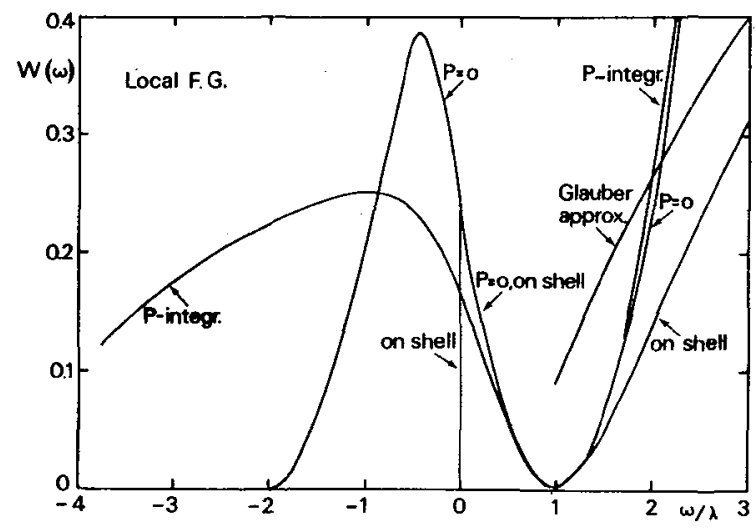

Fig. 3 - The imaginary part of the optical potential at $\mathrm{R}=0$ for various values of $\mathrm{p}$ and in the Glauber approximation.

$\delta$-function. One sees that the local equivalent and $P=0$ values are rather similar for positive energies but that the on shell value differs drastically for large energies. The Glauber approximation lies always above by virtue of the additional phase space gained by neglecting the Pauli principle.

In turning to finite size effects, $W$ can be calculated analytically on shell, $w=p^{2} / 2 m+V(R)$,

$$
W\left(\omega, 0 . s_{,}, R\right) \propto\left\{\begin{array}{ll}
0 & 0 \leq \omega \leq \lambda-\varepsilon_{f}(R) \\
\frac{1}{4}\left(p_{f}^{2}(R)-P^{2}\right)^{2} & \lambda-\varepsilon_{f}(R) \leq \omega \leq \lambda \\
\frac{2}{15}\left(2 p_{f}^{2}(R)-P^{2}\right)^{5 / 2} / P+\frac{1}{3} p_{f}^{3}(R) P-\frac{7}{15} P_{f}^{5}(R) / P & \lambda \leq \omega \leq \lambda+\varepsilon_{f}(R) \\
\frac{1}{3} p_{f}^{3}(R) P-\frac{7}{15} p_{f}^{5}(R) / P & \lambda+\varepsilon_{f}(R) \leq \omega<\infty .
\end{array} .\right.
$$

The dependence on $\mathrm{P}^{2}$ here is exactly the same as in infinite nuclear matter /2,10/ but with $\lambda$ replaced by the local $\varepsilon_{F}(R)$. However, eq. (16) is not strictly the same result as one would employ the local density approximation, i.e. replacing $\lambda$ by $\varepsilon_{F}(R)$ everywhere since the regions of validity of the different branches do depend on the absolute Fermi energy $\lambda$ as well.

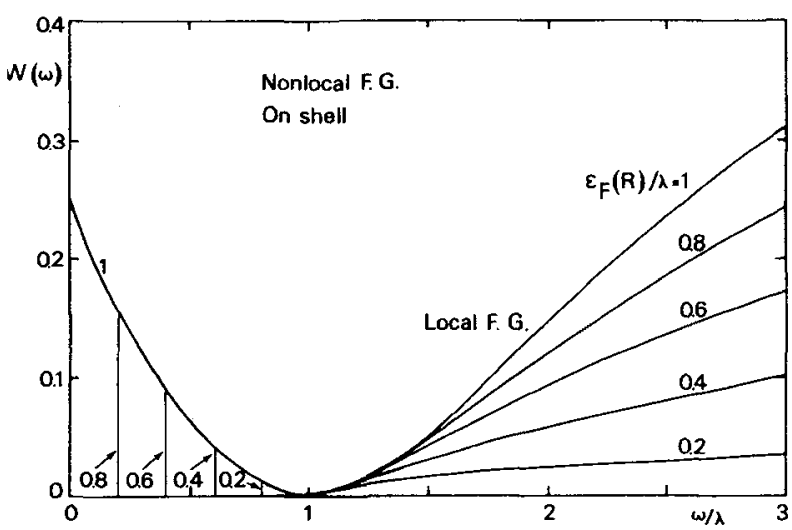

Fig. 4 - The imaginary part of the on shell optical potential for different values of the local Fermi energy. 
Fig. 4 shows the dependence of $W$ on $\varepsilon_{F}(R)$ or, in other words, on the radius $R$. The upper curve then corresponds to the nuclear interior and the lower ones to the surface. In assuming for instance a Woods-Saxon potential, for fixed energy, $W$ is almost constant in the interior and falls off sharply at the surface. The local density approximation, on the contrary, would yield curves shifted to the left and, thus, violating the basic feature of $W(\omega=\lambda, P, R)=0$.

\section{IV - FINITE RANGE FORCE}

Gaussian two-body effective interactions have typical ranges of $2.25 \mathrm{fm} / 14 /$, i.e. $\mathrm{k}_{\mathrm{o}} / \mathrm{k}_{\mathrm{F}}=0.625$. We therefore solved eq. (12) numerically for various ranges. The Gaussian exp $\left(-2 q^{2} / k_{o}{ }^{2}\right)$ cuts off effectively the high momentum transfer contributions and, hence, forces $W$ to decrease at high energy as shown in $W(\omega, 0 . s,, R=0)$ of Fig. 5. The strength of the force, here again, has been adjusted to attain

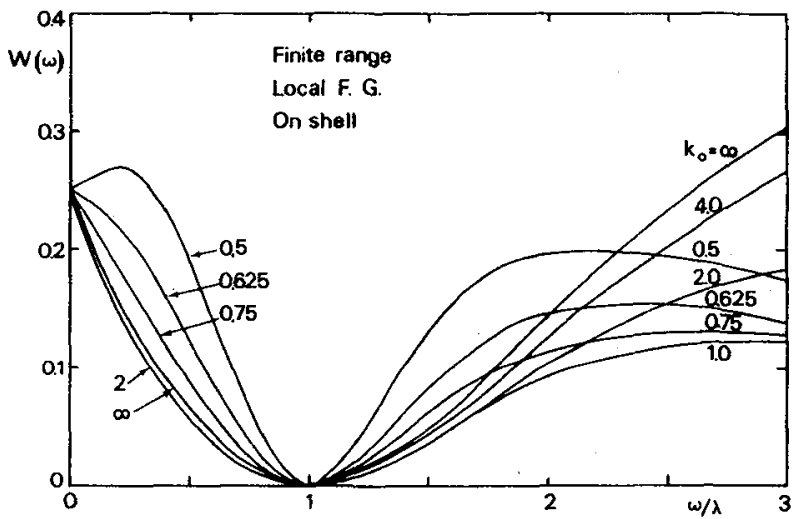

Fig. 5 - Imaginary part of the optical potential on shell at $\mathrm{R}=\mathrm{O}$ for various ranges $\mathrm{k}_{\mathrm{o}} / \mathrm{k}_{\mathrm{F}}$ of the two-body effective Gaussian interaction.

$W(\omega=0, P=0, R=0)=1 / 4 \lambda$. The larger the range, i.e. the smaller $k_{0}$ is, the more concentrated is $W$ around small energies.

For finite radii, $W$ now depends also on the nuclear mass number via $R_{\text {on }}$ ine $R^{\prime}-$ integration. Fig. 6 therefore shows $W\left(\omega, 0 . s\right.$, , R) for a ${ }^{40} \mathrm{Ca}$ harmonic oscillator and $k_{o} / k_{F}=0.625$ at various $\varepsilon_{F}(R)$. A comparison with the zero range equivalent,

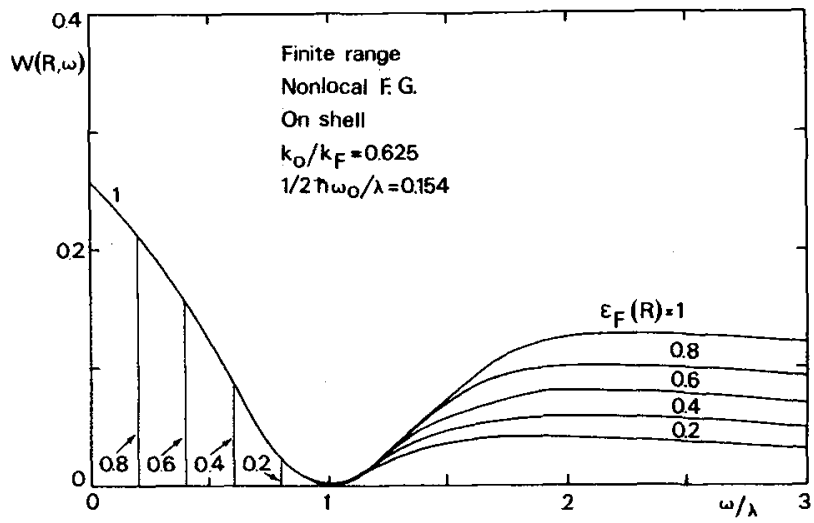

Fig. 6 - Imaginary part of the on shell optical potential for ${ }^{40} \mathrm{Ca}$ and various local Fermi energies.

Fig. 4, shows again the decrease of the optical potential in the surface and a concentration near small positive and negative energies.

Finally, we compare our exact calculations for finite range with those obtained by use of the Glauber approximation in Fig. 7. Here again, the additional false 


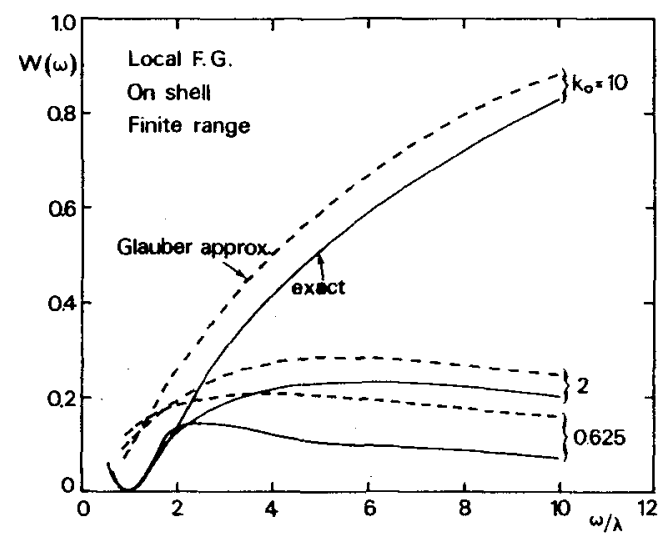

Fig. 7 - Imaginary part of the on shel1 optical potential at $\mathrm{R}=0$ and range $\mathrm{k}_{\mathrm{o}} / \mathrm{k}_{\mathrm{F}}=0.625$ compared with the Glauber approximation.

phase space gained by the neglection of the Pauli principle enhances $W$ roughly by a constant amount for all energies.

\section{SUMMARY AND OUTLOOK}

We have applied semiclassical methods for the calculation of the imaginary part of the optical potential $W(\omega, P, R)$. Finite size effects have been incorporated by means of the nonlocal Fermi gas approximation and a finite range effective interaction. As results we found that the finite range of the force is responsible for the fall off of $\mathrm{W}$ at high energies; that the nonlocality of $\mathrm{W}$ is strongly pronounced especially for small $\mathrm{P}$; that the Glauber approximation yields values of $\mathrm{W}$ always too high by about a constant amount and that the strict local density approximation only roughly simulates finite size effects.

Further studies on this subject will be devoted to the calculations of the real part of the optical potential via subtracted dispersion relations, thus obtaining e.g. the correction to the Hartree-Fock potential and realistic level densities around the Fermi energy, effective masses and momentum distributions.

\section{REFERENCES}

/1/ MAHAUX C. and NGỐ H., Phys, Lett. 10OB (198I) 285.

12/ ORLAND H. and SCHAEFFER R., Nuc1. Phys. A299 (1978) 442.

13/ SARTOR R. and MAHAUX C., Phys. Rev. C21 (1980) 1546.

14/ BERNARD V. and MAHAUX C., Phys. Rev. C23 (1981) 888.

15/ MAHAUX C. and NGô H., Nuc1. Phys. A410 (1983) 271.

16/ NEGELE J.W. and YAZAKI K., Phys, Rev. Lett. 47 (1981) 71.

/7/ MAHAUX C., Phys. Rev. C28 (1983) 1848.

18/ BROWN G. in "Many Body Problems" (North-Holland/Elsevier, Ansterdam, 1972) ch. 29.

19/ GALITSKII V.M., Zh. Eksp. Teor. Hiz. 34 (1958) 15] [Sov. Phys. IETP 7 (1958) $104]$.

/10/ BRENIG W., Nucl. Phys. 13 (1959) 333.

$111 /$ JEUKENNE J.P., LEJEUNE $\bar{A}$. and MAHAUX C., Phys. Rep. 25 (1976) 83.

112/ HASSE R.W. and SCHUCK P., Proc. Int. Conf. on Nuclear Physics, Florence, 1983 (Tipografia Compositori, Bologna, 1983) p. 777.

/13/ HASSE R.W. and SCHUCK P., Proc. Int. Symp. on Highly Excited States and Nuclear Structure, Orsay, 1983 (Orsay, 1983) p. 16.

/14/ GOGNY D. and PADJEN R., Nucl. Phys. A293 (1977) 365. 